\title{
Are We Ready for Real-Time Applications of Clinical NGS?
}

\section{Debmalya Barh $^{1 *}$, Marianna E. Ivanova ${ }^{2}$ and Vasco Azevedo}

${ }^{1}$ Centre for Genomics and Applied Gene Technology, Institute of Integrative Omics and Applied Biotechnology (IIOAB), Nonakuri, Purba Medinipur, WB-721172, India ${ }^{2}$ Oftalmic LLC., Bardina str. 4, Moscow 119334, Russia

${ }^{3}$ Laboratorio de Genetica Celular e Molecular, Departamento de Biologia Geral, Instituto de Ciencias Biologics, Universidade Federal de Minas Gerais CP 486, CEP 1270-901 Belo Horizonte, Minas Gerais, Brazil

The $\$ 1000$ genome concept is going to be reality soon due to the huge reduction in sequencing cost using the latest Next-Generation Sequencing (NGS) platforms. Current Illumina HiSeq / MiSeq and Ion Torrent platforms from Thermo Fisher Scientific Inc. have made significance contributions towards reducing the cost and increasing the speed of sequencing. The MinION USB stick DNA sequencer by Oxford Nanopore Technologies may be a key market player in near future considering cost, speed, and efficacy. On the other hand, cloudbased several bioinformatics platforms are in the market to analyse these NGS data. However, the quality of their data analysis and interpretation for clinical genome or exome is doubtful.

Several advantages of clinical NGS are recently found regarding rare disease diagnosis, risk and recurrence prediction, genetic predisposition, and drug responses among others. The currently available NGS technology platforms only detect SNVs, CNVs, small indels, etc. However, none of the sequencing and analysis platforms detect some of the important DNA variations such as repeat sequences, large deletions or duplications, translocations, inversions, etc. that are key diagnostic feature in several important diseases that include neurological disorders and cancers. Similarly, detection of a large number of false positive or negative variants, variants of unknown significance, and efficient pharmacogenomics translation of the sequences are real problems at this current scenario. Furthermore, the current technologies require long Turned Around Time (TAT) and cannot offer affordable point-of-care DNA-based diagnostics to the common people. Hence, the up-to-date clinical NGS is limited to specific diseases and needs much more advancements to be used for decision making and patient specific disease management by the clinicians. However, targeted NGS panels have advantages over clinical exome and whole genome sequencing: TAT is much faster and the interpretation of results obtained is much more acceptable, since only known and proven pathogenic mutations are considered in the panels.

On the other hand, while strict regulatory guidelines in each step of genetic test or clinical NGS-based test are followed in the USA or some of the European countries; there are no such regulatory bindings found in many developing and underdeveloped countries. Also, due to dilution of DNA/Gene patents; several companies are introducing in the molecular diagnostic market to provide genetic tests using NGS technologies. It could be alarming if a combination of the lack of standard guidelines, in situ issues of NGS, and unskilled manpower handles such cutting-edge evolving technology to provide genetic tests directly to the patients. It will not help the doctors to make the decision for their patients; Instead the patients have to spend massive cost for the tests, and the benefits will go to the test provider companies.

As per our opinion, the clinical NGS needs long run to reach to patients' bedside. While the whole genome or exome-based NGS is worthy for clinical research and to develop the genotype-phenotype knowledge base, they may not be used for real-time clinical practice at this moment. The TAT needs to be very fast, and all tests should follow proper regulatory guidelines. Targeted NGS to detect known variations having reported and well established clinical or pharmacogenetic interpretations are recommended for today's patient care.
*Corresponding author: Debmalya Barh, Centre for Genomics and Applied Gene Technology, Institute of Integrative Omics and Applied Biotechnology (IIOAB), Nonakuri, Purba Medinipur, WB-721172, India, Tel: +91 944955 0032; E-mail: dr.barh@gmail.com

Received Novmber 03, 2015; Accepted Novmber 05, 2015; Published Novmber 09,2015

Citation: Barh D, Marianna E. Ivanova, Azevedo V (2015) Are We Ready for Real-Time Applications of Clinical NGS?. Next Generat Sequenc \& Applic 2: 122 doi:10.4172/2469-9853.1000122

Copyright: $\odot 2015$ Barh D, et al. This is an open-access article distributed unde the terms of the Creative Commons Attribution License, which permits unrestricted use, distribution, and reproduction in any medium, provided the original author and source are credited. 